\title{
Single-Balloon Enteroscopy-Guided ERCP in Surgically Altered Anatomy Is Safe and Highly Effective: Results from a Prospective Study
}

\author{
Partha Pal ${ }^{1}$ Sujay Ashok Kulkarni ${ }^{1} \quad$ Hrushikesh Chaudhari ${ }^{1}$ Mohan Ramchandani ${ }^{1}$ \\ P. Manohar Reddy ${ }^{1} \quad$ Pankaj Kumar Shrimal ${ }^{1} \quad$ D. Nageshwar Reddy ${ }^{1} \quad$ Manu Tandan $^{1}$
}

${ }^{1}$ Department of Medical Gastroenterology, Asian Institute of Gastroenterology, Hyderabad, India
Address for correspondence Partha Pal, MD, DNB, Department of Medical Gastroenterology, Asian Institute of Gastroenterology, Hyderabad 500082, India (e-mail: partha1986@yahoo.com).

\section{Abstract \\ Keywords \\ - single-balloon enteroscopy \\ - endoscopic retrograde cholangiopancreatog- raphy \\ - surgically altered anatomy}

Objectives Single-balloon enteroscopy (SBE)-assisted endoscopic retrograde cholangiopancreatography (ERCP) has been shown to be cost effective and less technically demanding than other modes of device-assisted enteroscopy. We aimed to evaluate the safety and efficacy of SBE-ERCP in surgically altered anatomy.

Methods This was a prospective cohort study in a high-volume tertiary care gastroenterology center. Patients with surgically altered anatomy requiring pancreaticobiliary interventions, who failed ERCP with a colonoscope/duodenoscope, underwent SBE-ERCP. Diagnostic and therapeutic success, procedure time, and adverse events were evaluated prospectively with short-term follow-up.

Results Twenty-eight patients with surgically altered anatomy (19 males, aged 15-66 years) underwent total 40 SBE-ERCP (23 Roux-en-Y, 17 Billroth II anatomy) procedures. The ERCP diagnosis were hepaticojejunostomy $(H J)$ stricture $(N=10)$, HJ stricture and stone $(N=7)$, common bile duct $(C B D)$ stricture $(N=6)$, CBD stone $(N=1)$, pancreaticojejunostomy (PJ) stricture $(N=1)$, and pancreatic duct $(P D)$ stricture $(N=1)$. Diagnostic success was achieved in $91.3 \%(21 / 23)$ patients with Roux-en-Y anatomy and $100 \%$ (17/17) with Billroth II anatomy. Therapeutic success was achieved in $86.95 \%$ (20/23) and 94.1\% (16/17) patients with Roux-en-Y and Billroth II anatomy, respectively. In patients with intact papilla $(N=8)$, diagnostic and therapeutic success were achieved in $100 \%(8 / 8)$ and $75 \%(6 / 8)$ cases, respectively. The mean procedure time for Roux-en-Y and Billroth II anatomy were 64.95 minutes (range 30-110 min) and 38.31 minutes (range 25-60 min), respectively. Immediate complications occurred in $7.5 \%$ (3/40) procedures ( 2 requiring laparotomy, 1 treated endoscopically) in the form of perforation. Among delayed complications, 5 patients had cholangitis and 1 had recurrent cholestasis, which were successfully treated with stent exchange. No other complications occurred over median follow-up of 110 days (30-390 d).

Conclusion SBE-ERCP in surgically altered anatomy requiring pancreatic-biliary intervention appears safe and highly effective with current long ERCP accessories. Further improvement in SBE-ERCP technique and accessories has the potential to make it a promising avenue in surgically altered anatomy.
DOI https://doi.org/ 10.1055/s-0040-1709814 ISSN 0976-5042.
(C)2020 Society of Gastrointestinal Endoscopy of India
License terms

(요 (1) $\ominus \circledast$ 


\section{Introduction}

Endoscopic retrograde cholangiopancreatography (ERCP) is the accepted therapeutic modality for management of many biliopancreatic disorders. ERCP is successful in near 90\% of patients with normal anatomy. ${ }^{1}$ In the recent times, aggressive pancreaticobiliary and bariatric surgery are performed regularly resulting in altered gastrointestinal anatomy. ERCP in a surgically altered anatomy is a technical challenge. This is because of the long afferent loop in a Billroth II gastrectomy. In a Rouxen-Y reconstruction, both afferent and efferent loops are long and often an acute angulation at the junction of the anastomotic limbs makes approach to the papilla difficult. Colonoscopes have been used to overcome this limitation. ${ }^{2}$ Percutaneous transhepatic cholangiography (PTC) has also been utilized in selected patients for biliary intervention. PTC, however, has its own limitation and cannot be used for pancreatic cannulation. ${ }^{3}$ Surgery then remains the only other alternative in patients with altered anatomy. ${ }^{4}$ Surgery is, however, associated with longer hospitalization, increased morbidity, and higher costs.

Device-assisted enteroscopy (DAE) have been used successfully over the past decade for biliary and pancreatic cannulation in patients with surgically altered anatomy. ${ }^{5-7}$ However, most case series have small cohort of patients.

In this study we describe our experience of use of SBE, a device-assisted enteroscope (DAE) in performing ERCP in patients with altered surgical anatomy.

\section{Materials and Methods}

The aim of the study was to evaluate safety and efficacy of SBE-assisted ERCP in patients with surgically altered anatomy (Billroth II and Roux-en-Y).

The study was conducted between March 2016 and February 2017 at Asian Institute of Gastroenterology, Hyderabad, a high-volume tertiary care center for gastroenterology. This is a prospective cohort study approved by institutional review board and informed consent was taken from all the patients prior to enrolment.

Patients with surgically altered anatomy who previously had successful ERCP with a colonoscope or duodenoscope were excluded. Twenty-eight patients (19 males) with a median age of 48 years (range 15-66 y) underwent total 40 SBE-ERCP procedures. All the patients had prior history of surgical interventions and hence altered anatomy; 12 patients had history of Roux-en-Y hepaticojejunostomy (HJ), 8 had history of Whipple's pancreatic duct (PD) (4 had RouX-en-Y biliary reconstruction-RY-BR, and 4 had classic Whipple's PD), 7 had Billroth II gastrectomy, and 1 had total gastrectomy with Roux-en-Y esophagojejunostomy TG (total gastrectomy) + EJ (esophagojejunostomy).

Indications of SBE-ERCP procedures were cholangitis $(N=22)$, obstructive jaundice $(N=4)$, and pancreatic endotherapy $(N=2)$. Repeat procedure $(N=12)$ indications were cholangitis $(N=5)$, obstructive jaundice due to blocked stent $(N=1)$, stent exchange after pancreatic endotherapy $(N=2)$, and follow-up stent removal $(N=4)$ ( - Table $\mathbf{1})$.

For purpose of discussion we divided the cases according to the anatomy into two categories-(1) Roux-en-Y (RY) anatomy $(N=17$, RY-HJ, $N=11$; RY-BR in Whipple's PD, $N=4$; TG + EJ, $N=1)$ and (2) Billroth II anatomy ( $N=11$, Billroth II GJ, $N=7$; classical Whipple's PD, $N=4$ ). Patients with classical Whipple's PD were included in the second group as anatomically and endoscopically they have similar gastrojejunostomy and relatively short afferent limb. Similarly, patients with Whipple's PD with RY-BR were included in the first group.

All patients with RY anatomy underwent SBE-ERCP directly without any prior ERCP, whereas all patients with Billroth II reconstruction underwent ERCP with standard duodenoscope/pediatric colonoscope initially, failing which they were subjected to SBE-ERCP.

\section{SBE Instruments}

The SBE instruments consisted of a videoenteroscope (XSIFQ260Y; Olympus Medical Systems, Tokyo, Japan), a sliding tube with a balloon (XST-SB1; Olympus), and a balloon controller (XMAJ- 1725; Olympus). The working length of the videoenteroscope was $200 \mathrm{~cm}$, with an outer and working channel diameter of $9.2 \mathrm{~mm}$ and $2.8 \mathrm{~mm}$, respectively. The sliding tube with a silicone balloon at its tip has a working length, and outer and inner diameters of $132 \mathrm{~cm}, 13.2 \mathrm{~mm}$, and $11 \mathrm{~mm}$, respectively. ${ }^{6}$

\section{SBE-ERCP Procedure}

All procedures were performed by experienced pancreaticobiliary endoscopist (H.C. and M.R.) under monitored anesthesia, with patient in prone/semiprone position.

The enteroscope was advanced using a push and pull technique, with sequential inflation and deflation of balloon. The enteroscope was inserted as far as possible in a deflated position, the balloon was inflated, and then the entire system was withdrawn to promote pleating of the small bowel onto the outer surface of the overture. Then the enteroscope was advanced with the balloon inflated, holding the small bowel in retracted position. Fluoroscopy was used in all the cases to guide the passage of the enteroscope to the ampulla or $\mathrm{HJ}$ site and to perform ERCP.

Endoscopic accessories for SBE-ERCP were all commercially available. It was predecided that if deep cannulation could not be achieved despite repeated attempts using various techniques for 60 minutes, the procedure would be terminated. On failure of cannulation, we scheduled percutaneous transhepatic biliary drainage (PTBD) under transabdominal ultrasound guidance by an expert interventional radiologist. Prophylactic antibiotics were administered prior to all procedures in all cases. No patients received NSAIDs, antiplatelet agents, or antithrombotic agents. The outcomes were evaluated prospectively regarding success rate, therapeutic procedure, and complications, as well as short-term follow-up.

\section{Definitions of Treatment Outcomes}

Endoscopic success was defined as ability to intubate the afferent limb and identify papilla or pancreatoenteric/bilioenteric anastomotic site. Diagnostic success was defined as 
successful duct cannulation and cholangiogram leading to a diagnosis. Therapeutic ERCP success was defined as a successful diagnostic enteroscopy with successful therapeutic interventions. Total procedure time was defined as the time from the beginning of insertion of enteroscope to complete withdrawal.

\section{Complications}

Bleeding was considered an adverse event when it required therapeutic intervention at the time of procedure or identified later requiring blood transfusion or hospitalization. Perforation was discovered by presence of free intra-abdominal air on imaging studies. Cholangitis was defined as per Tokyo guidelines and post-ERCP pancreatitis was defined by the criteria given by Cotton et al. ${ }^{8,9}$

\section{Results}

Total number of procedures in RY anatomy was 23 SBE-ERCP (initially 17 and 6 repeat procedures) and in Billroth II anatomy was 17 procedures (initially 11 and 6 repeat procedures). In RY anatomy, diagnostic success was achieved in 21/23 patients (91.3\%) and $\mathrm{HJ}$ site could not be reached in 2 patients who had bowel perforation due to difficult anatomy with long Roux limb and interloop bowel adhesions found later on laparotomy. In the Billroth II anatomy, all patients had diagnostic success $(100 \%, 17 / 17)$ (-Table $\mathbf{1})$.

The ERCP diagnosis were HJ stricture $(N=10)$, HJ stricture and stone $(N=7)$, common bile duct (CBD) stricture $(N=6)$, CBD stone $(N=1)$, pancreaticojejunostomy (PJ) stricture $(N=1)$, and PD stricture $(N=1)(-$ Table 2$)$.
Among the patients who had diagnostic success, two had therapeutic failure due to tight stricture defying passage of accessories, one each in both groups (one in TG + EJ and one in Billroth II GJ) ( - Fig. 1 ).

HJ strictures alone $(N=10)$ were treated with balloon

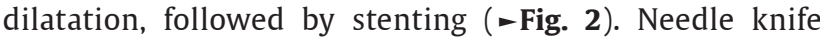
stricturoplasty was done for tight $\mathrm{HJ}$ stricture in 2 patients among which one had perforation which was managed successfully with enteroscopy-assisted clipping. HJ stricture with stones $(N=7)$ were treated similarly with addition of stone clearance. On follow-up, 4 patients developed cholangitis, requiring stent exchange. Stent was removed in two patients after resolution of stricture.

For CBD strictures $(N=6)$ and stones $(N=1)$, deep CBD cannulation was achieved by wire-guided technique or precut sphincterotomy, followed by biliary sphincterotomy. Later, CBD stenting was done for strictures and balloon sweep and clearance was done for CBD stones. In spite of deep biliary cannulation and cholangiogram, therapeutic intervention failed in two cases due to tight stricture (1 in Billroth II GJ anatomy and 1 in TG + EJ) ( - Fig. 3).

Pancreatic endotherapy (PD stent and/or stricture dilatation) was done in two patients, (one PD stricture and PJ stricture each).

In patients with intact papilla ( $N=8$; Billroth II GJ, $N=7$; TG + EJ, $N=1), 4$ patients underwent biliary sphincterotomy and 1 patient underwent pancreatic sphincterotomy. Precut sphincterotomy with needle knife was done in two patients. In rest of the two patients, biliary instrumentation failed.

The mean procedure time for RY anatomy was 64.95 minutes (range 30-110 min) and for Billroth II anatomy was 38.31 minutes (range 25-60 min). Among Billroth II cases, the mean procedural time for those with intact papilla

Table 1 Enteroscopy diagnostic and therapeutic success rates in various subgroups

\begin{tabular}{|l|l|l|l|}
\hline & $\begin{array}{l}\text { Enteroscopy } \\
\text { success }\end{array}$ & $\begin{array}{l}\text { Diagnostic } \\
\text { success }\end{array}$ & $\begin{array}{l}\text { Therapeutic } \\
\text { success }\end{array}$ \\
\hline $\begin{array}{l}\text { Billroth II anatomy (BII GJ/Classic Whipple's) } \\
(N=11+6 \text { repeat procedures) }\end{array}$ & $100 \%(17 / 17)$ & $100 \%(17 / 17)$ & $94.1 \%(16 / 17)$ \\
\hline RY-BR $(N=17+6$ repeat procedures) & $91.3 \%(21 / 23)$ & $91.3 \%(21 / 23)$ & $86.95 \%(20 / 23)$ \\
\hline Overall & $95 \%(38 / 40)$ & $95 \%(38 / 40)$ & $90 \%(30 / 40)$ \\
\hline
\end{tabular}

Abbreviations: BII, Billroth II; EJ, esophagojejunostomy; G], gastrojejunostomy; RY-BR, Roux-en-Y biliary reconstruction; TG, total gastrectomy.

Table 2 Details of results of single-balloon enteroscopy-guided endoscopic retrograde cholangiopancreatography (ERCP) and therapeutic procedures performed

\begin{tabular}{|l|l|}
\hline ERCP diagnosis $(\mathbf{2 6 / 2 8 )}$ & Therapeutic ERCP \\
\hline HJ stricture, $N=10$ & HJ stenting $(N=14$, repeat $N=4)$ \\
\hline HJ stricture with stone, $N=7$ & HJ balloon dilatation $(N=13$, repeat, $N=4)$ \\
\hline & HJ balloon sweep $(N=6)$ \\
\hline CBD stricture, $N=6$ & CBD stenting $(N=4$, repeat $N=2)$ \\
\hline CBD stone, $N=1$ & CBD balloon sweep $(N=1)$ \\
\hline PJ stricture, $N=1$ & PD/PJ stenting $(N=2$, repeat $N=2)$, \\
\hline PD stricture, $N=1$ & PJ balloon dilatation $(N=1)$ \\
\hline
\end{tabular}

Abbreviations: BII, Billroth II; CBD, common bile duct; EJ, esophagojejunostomy; GJ, gastrojejunostomy; HJ, hepaticojejunostomy; PD, pancreatic duct; PJ, pancreaticojejunostomy; RY-BR, Roux-en-Y biliary reconstruction; TG, total gastrectomy. 


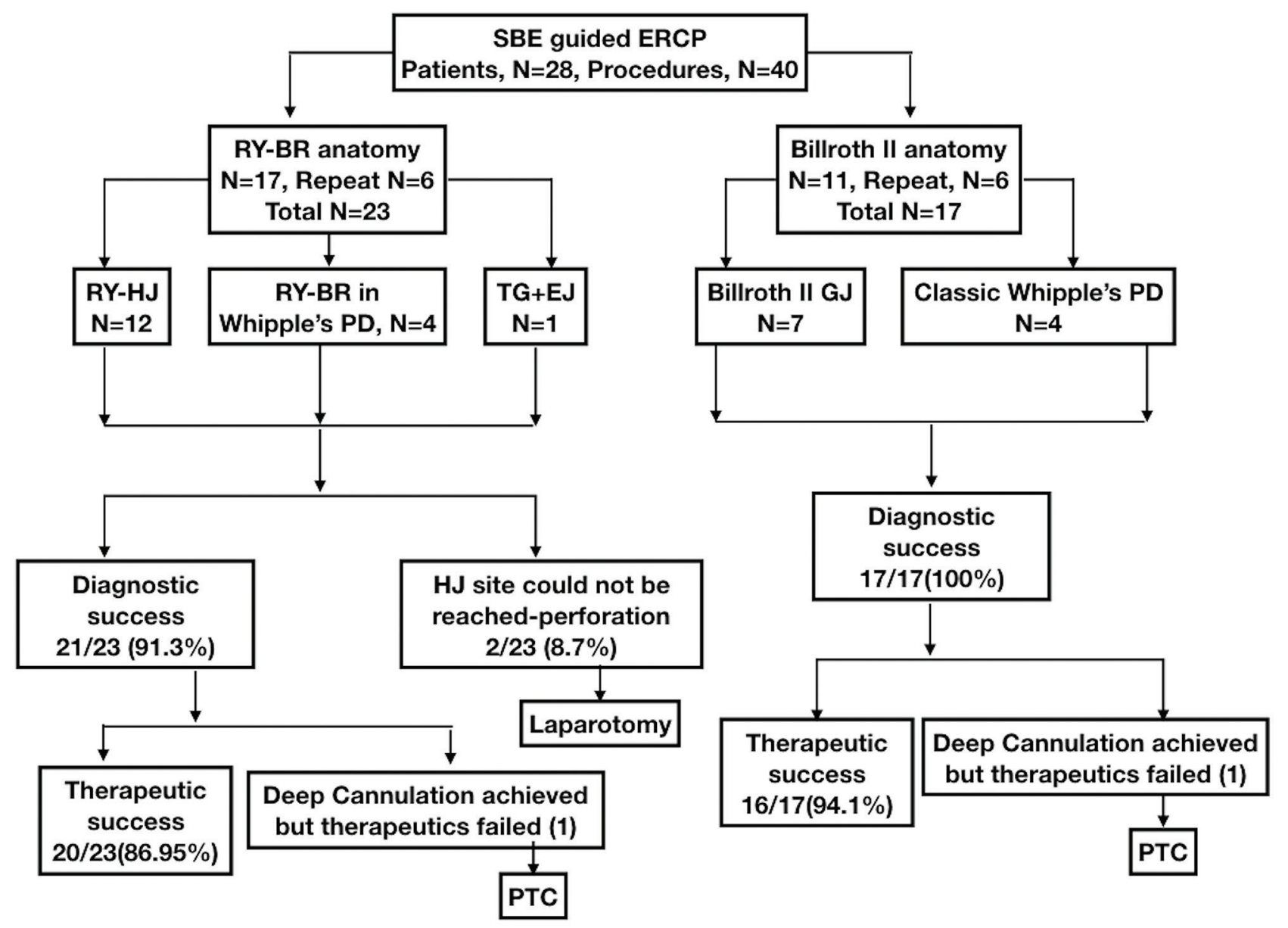

Fig. 1 Flowchart showing results of single-balloon enteroscopy (SBE)-assisted endoscopic retrograde cholangiopancreatography (ERCP) stratified according to different altered anatomy. EJ, esophagojejunostomy; GJ, gastrojejunostomy; PD, pancreaticoduodenectomy; PTC, percutaneous transhepatic cholangiography; RY-BR, Roux-en-Y biliary reconstruction; RY-HJ, Roux-en-Y hepaticojejunostomy; TG, total gastrectomy.

(Billroth II GJ) was 37.11 minutes (range 25-60 min) and for those with bilioenteric anastomosis was 39.85 minutes (range 28-60 $\mathrm{min}$ ).

Immediate complications occurred in 7.5\% (3/40) procedures in the form of perforation. In 2 cases requiring laparotomy which showed dense adhesions and 1 case (during needle knife stricturoplasty of tight HJ stricture) was successfully treated endoscopically by clipping. There were no cases of major bleeding during SBE-ERCP.

Among delayed complications, 5 patients had cholangitis and 1 had jaundice after initial ERCP, which was successfully treated with stent exchange. No other complications occurred over a median follow-up of 110 days (30-390 d).

\section{Discussion}

The modern obesity epidemic has increased the number of surgical procedures like RouX-en-Y gastric bypass (RYGB) which is complicated by formation of cholesterol gallstones in one-third of patients due to rapid weight loss and hence increase the need for biliary interventions. ${ }^{10,11}$ Moreover, increasing number of patients undergoing liver transplantation with Roux-en-Y biliary reconstruction (RYBR) due to postoperative adverse events or diseased bile ducts are at risk of biliary adverse events requiring billiard intervention in altered anatomy. ${ }^{12}$ Double-balloon, single-balloon, and spiral enteroscopes are the current modalities of device-assisted enteroscopy (DAE). Double-balloon enteroscopy (DBE) requires specialized equipment and expertise, which is not widely available. Spiral enteroscopy is still evolving. ${ }^{7}$ Singleballoon enteroscopy (SBE) uses single-balloon splinting overtube, which reduces and pleats small intestine, which help negotiate acute angulations in altered anatomy. SBE has shorter procedure time and less technically demanding than DBE with similar rates of adverse events. ${ }^{6,7,13}$ Failure of achieving the objective can occur in 30 to $40 \%$ cases with SBE and 20\% with DBE. ${ }^{10,14}$ However, given the reasonable success rate, good safety profile, and availability, SBE has the potential to be used as first line for ERCP in altered anatomy in which standard duodenoscope or colonoscope has failed..$^{15}$

Our present study demonstrates the safety and efficacy of SBE-ERCP in patients with altered surgical anatomy (in both Billroth II and RY anatomy) in a cohort of 28 patients undergoing 40 SBE-ERCP procedures. These included 11 patients with Billroth II anatomy and17 patients with Roux-en-Y anatomy. The endoscopic success rate in Billroth II (which includes 


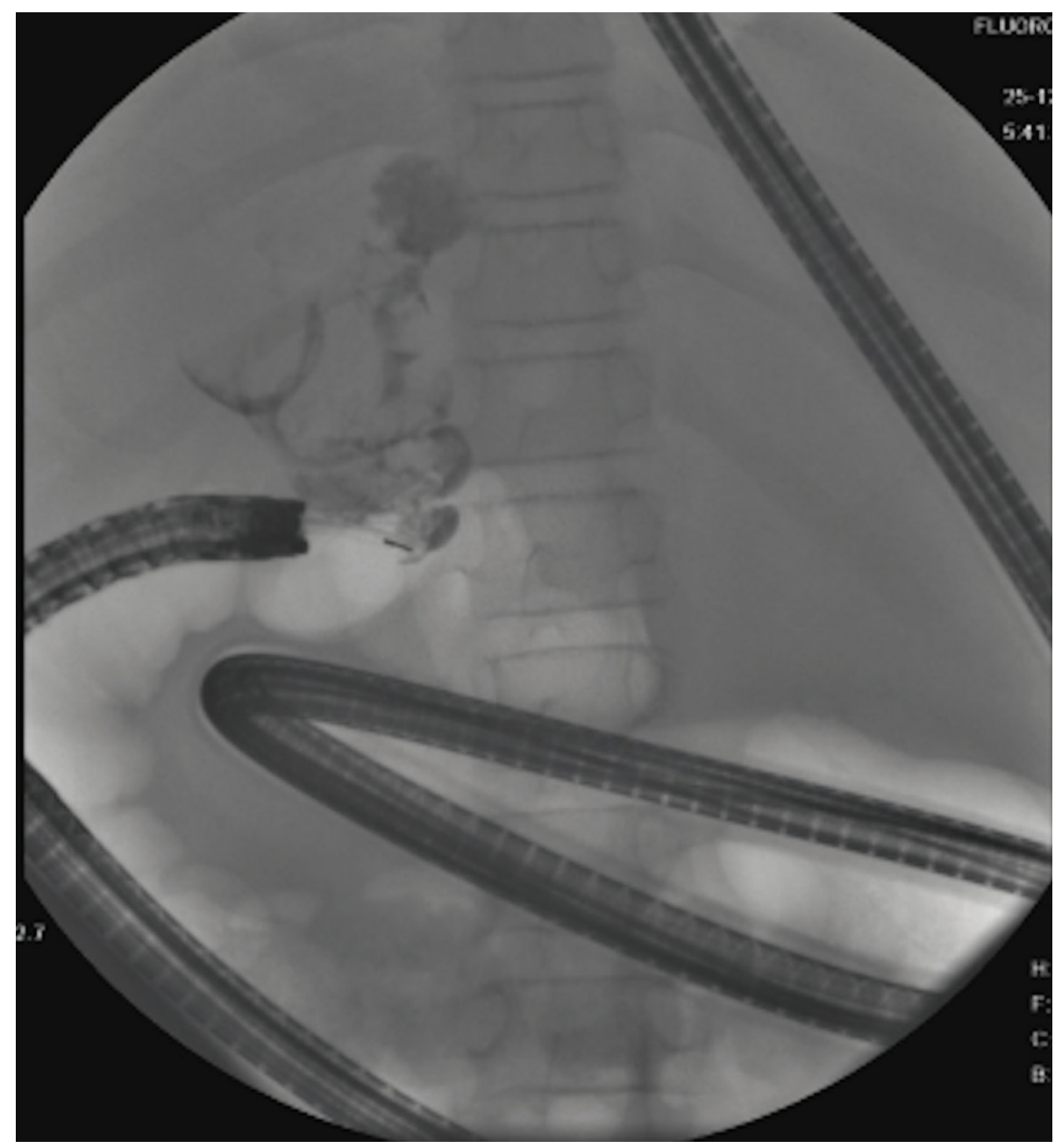

Fig. 2 Single-balloon enteroscopy (SBE)-guided endoscopic retrograde cholangiopancreatography (ERCP) for hepaticojejunostomy (HJ) stricture dilatation following choledochal cyst excision. SBE was passed up to the HJ site through Roux-en-Y limb and cholangiogram showed HJ structure with intrahepatic calculi. The HJ site was subsequently dilated and biliary stents were placed across it.

Billroth II GJ and classical Whipple's PD) and Roux-en-Y anatomy (RY-HJ and RY-BR in Whipple's PD) were $100 \%$ and $91.3 \%$, respectively. The higher success rate in Billroth II reconstruction is due to the fact that it has short afferent limb $(40-60 \mathrm{~cm}$ in case of classic Whipple's PD and even shorter in B II GJ). The lower success rate in RouX-en-Y anatomy is due to longer afferent limb (20-75 cm in case of RY-HJ and even longer with RY-BR in Whipple's PD which resembles Roux-en-Y gastric bypass [RYGB]), small bowel adhesions and acute angulation at the Y limb. Diagnostic success was similar in both the groups. Therapeutic success was $94.1 \%$ and $86.95 \%$ in BII and RY anatomy, respectively. The results are similar to recent studies on device-assisted enteroscopy-guided ERCP and significantly better than earlier studies due to commercially available long accessories for ERCP. ${ }^{6,16}$ In cases of intact papilla, SBE-ERCP is difficult due to oblique angulation of the papilla and poor maneuverability, leading to higher procedure time. ${ }^{6,17}$ In our entire cohort of 40 patients, out of the 4 failed cases, 2 had intact papilla (1 in BII GJ and 1 in TG + EJ) in which diagnostic cholangiogram was feasible but therapeutic intervention failed due to reasons mentioned earlier. The overall success rate was $75 \%$ in cases of intact papilla.
Earlier published reports on SBE-ERCP had lot of heterogeneity in various anatomy in which it was done. ${ }^{15} \mathrm{~A}$ meta-analysis by Inamdar et al reported endoscopic success, diagnostic success, and therapeutic success as $80.9 \%, 69.4 \%$, and $61.7 \%$, respectively. ${ }^{7}$ To adjust for the heterogeneity of the studies Abu Dayyeh et al showed in separate meta-analysis for various anatomies that showed respective diagnostic and therapeutic success rates of 75\% and 63\% in RYGB and 79\% and 65\% in RY-BR/Whipple's PD, respectively. So, overall 60 to $70 \%$ success rate was expected. But these contain older studies in which SBE was replaced by conventional endoscope and was done with short accessories due to unavailability of long ERCP accessories..$^{15}$ A recent report by Itokawa et al shows higher diagnostic (89.3\% and $93.8 \%$ in $\mathrm{RY}-\mathrm{HJ}$ and Whipple's PD, respectively) and therapeutic success rates (90\% and 95\% in RY-HJ and Whipple's PD, respectively, includes data for both SBE, short SBE, and DBE). ${ }^{18}$ This is due to availability of longer ERCP accessories and larger working channel (3.2 mm) available with short SBE. With modern variations in SBE-ERCP instruments and available accessories, some of the difficulties are overcome. Modern short SBE (prototype SIF-Y0004-V01, 3.2-mm diameter working 


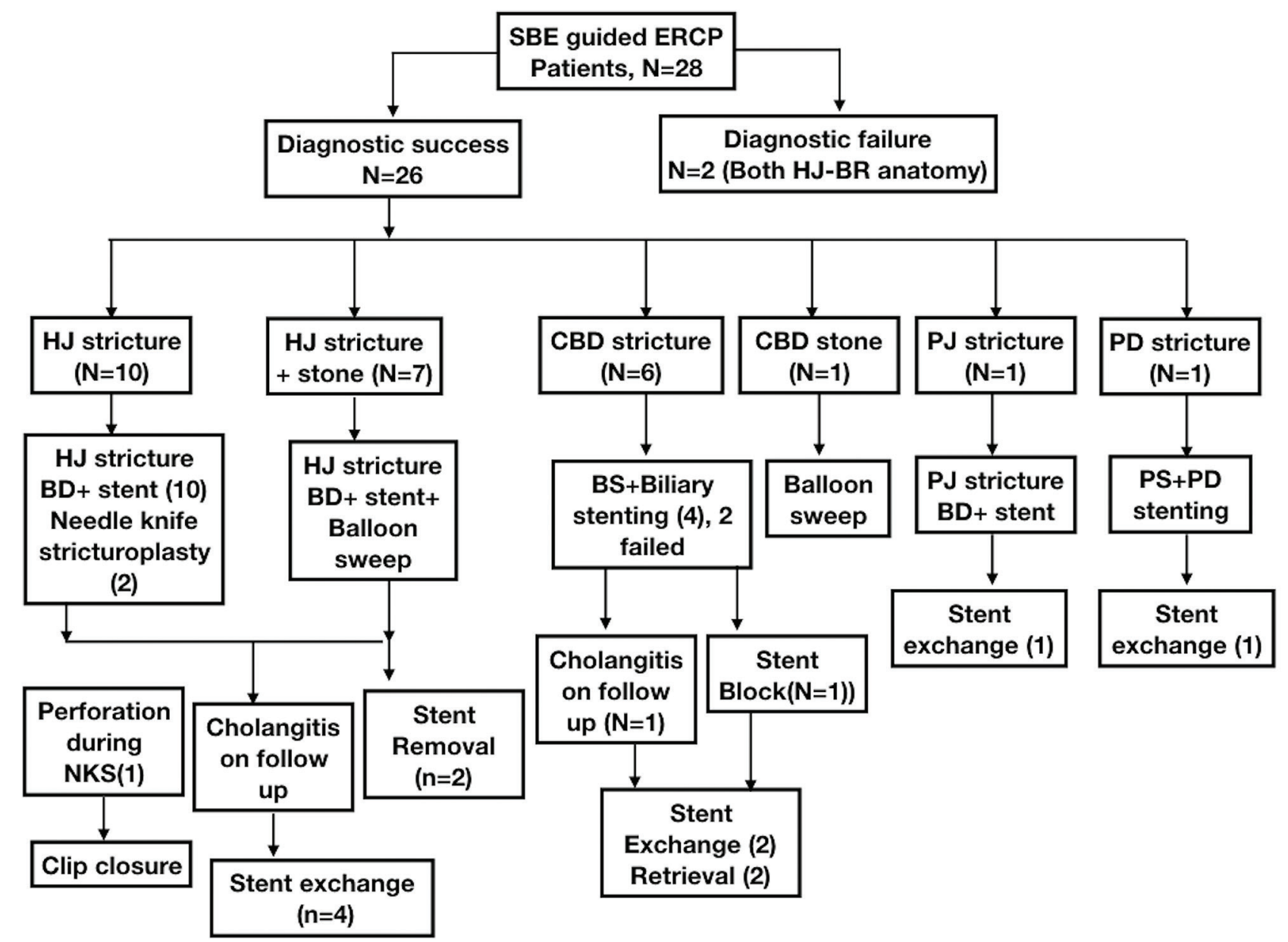

Fig. 3 Flowchart showing details of successful and failed single-balloon enteroscopy (SBE)-guided endoscopic retrograde cholangiopancreatography (ERCP). BD, biliary drainage; BS, biliary sphincterotomy; CBD, common bile duct; EJ, esophagojejunostomy; G], gastrojejunostomy; NKS, needle knife sphincterotomy; PD, pancreaticoduodenectomy; PTC, percutaneous transhepatic cholangiography; PJ, pancreaticojejunostomy; PD, pancreatic duct; RY-BR, Roux-en-Y biliary reconstruction; RY-HJ, Roux-en-Y hepaticojejunostomy; TG, total gastrectomy.

channel; Olympus) has $3.2 \mathrm{~mm}$ working channel allowing for introduction of basket catheter, intraductal ultrasound (IDUS) probe, and large balloon dilatation. Wider working channel enables swift 7 Fr stent placement even with the enteroscope in retroflexed position and better management of post sphincterotomy bleed if it occurs. ${ }^{19} \mathrm{~A}$ new therapeutic XSIF-180JY SBE device (a long-type SBE device: $200 \mathrm{~cm}$ ) has been described which has additional water jet channel which can be used to flush away biliary stones and blood without the need to clear the wider working channel $(3.2 \mathrm{~mm}) .^{20}$ Other important reasons for higher success rate is availability of long ERCP catheters, guide wires, and dilatation balloons which does not require exchange of SBE with conventional upper GI scope used in earlier studies with SBE-ERCP. ${ }^{16}$

ERCP in an altered anatomy is a challenge. The challenges in therapeutic endoscopy are similar in both RY and BII anatomy. As mentioned earlier, the long afferent limb in Billroth II gastrectomy and the length of both afferent and efferent limbs as well as sharp angulation in Roux-en-Y procedure, limit the success using standard ERCP and colonoscope. Yet another limitation is the lack of proper instrumentation for use through the SBE due to narrow working channel diameter. The absence of an elevator (as seen in the standard duodenoscope) is a handicap for the endoscopist. The elevator helps in both cannulation and deployment of accessories. The oblique position of the native papilla is another limitation in cannulation. ${ }^{6,18}$ In RY-HJ, the intact stomach allows looping of enteroscope, afferent limb is long as jejunojejunostomy is encountered distal to ligament of Treitz and anastomosis can be difficult to identify, especially if strictured. In classical Whipple's PD similar problem occur except for shorter afferent limb. The difference in therapeutic success rate is mainly due to lower diagnostic success in RY anatomy (in two cases HJ site could not be reached). DAE is successful for papillary cannulation if the length of the Roux limb is less than $150 \mathrm{~cm}$. Beyond $150 \mathrm{~cm}$, the success rate is limited and alternative techniques (e.g., endoscopic ultrasound-guided biliary cannulation) may have to be utilized. ${ }^{15,21,22}$ For Roux limb greater than $150 \mathrm{~cm}$, if DAE-assisted ERCP fails, EUSguided gastrogastrostomy creation followed by conventional ERCP can be done (EUS-GG-ERCP). ${ }^{21}$ Bukhari et $\mathrm{al}^{23}$ showed higher technical success, shorter procedure time, and similar 
safety profile with EUS-GG-ERCP in RYGB anatomy compared with DAE-assisted ERCP. ${ }^{24}$ However, due to lack of widespread availability of expertise, invasiveness, and cost factors involved, DAE-ERCP can be the preferred initial approach in postsurgical altered anatomy.

Complications like perforation was higher in RY anatomy due to longer afferent limbs allowing looping and due to adhesions. Complication rate was 7.5\% (3/40), which is comparable to PTBD which has a reported adverse event rate of $9 \%$ and a major adverse event rate of $4 \%{ }^{16,24}$

The limitations of the present study are case selection and treatment bias because of it being a retrospective, single-center study.

In conclusion, SBE-ERCP in patients with R-Y and BII anatomy seems to be safe and effective. Improvement in both the technique and technology will further improve the success rate.

\section{Conflict of Interest}

None declared.

\section{References}

1 Freeman ML, Guda NM. ERCP cannulation: a review of reported techniques. Gastrointest Endosc 2005;61(1):112-125

2 Chahal P, Baron TH, Topazian MD, Petersen BT, Levy MJ, Gostout CJ. Endoscopic retrograde cholangiopancreatography in post-Whipple patients. Endoscopy 2006;38(12):1241-1245

3 Maaser C, Lenze F, Bokemeyer $\mathrm{M}$, et al. Double balloon enteroscopy: a useful tool for diagnostic and therapeutic procedures in the pancreaticobiliary system. Am J Gastroenterol 2008;103(4):894-900

4 Teplick SK, Flick P, Brandon JC. Transhepatic cholangiography in patients with suspected biliary disease and nondilated intrahepatic bile ducts. Gastrointest Radiol 1991;16(3):193-197

5 Shimatani M, Takaoka M, Tokuhara M, Miyoshi H, Ikeura T, Okazaki K. Review of diagnostic and therapeutic endoscopic retrograde cholangiopancreatography using several endoscopic methods in patients with surgically altered gastrointestinal anatomy. World J Gastrointest Endosc 2015;7(6):617-627

6 Itoi T, Ishii K, Sofuni A, et al. Single-balloon enteroscopy-assisted ERCP in patients with Billroth II gastrectomy or Roux-en-Y anastomosis (with video). Am J Gastroenterol 2010;105(1):93-99

7 Inamdar S, Slattery E, Sejpal DV, et al. Systematic review and meta-analysis of single-balloon enteroscopy-assisted ERCP in patients with surgically altered GI anatomy. Gastrointest Endosc 2015;82(1):9-19

8 Cotton PB, Lehman G, Vennes J, et al. Endoscopic sphincterotomy complications and their management: an attempt at consensus. Gastrointest Endosc 1991;37(3):383-393

9 Kiriyama S, Takada T, Strasberg SM, et al; Tokyo Guidelines Revision Committee. New diagnostic criteria and severity assessment of acute cholangitis in revised Tokyo Guidelines. J Hepatobiliary Pancreat Sci 2012;19(5):548-556

10 Azeem N, Tabibian JH, Baron TH, et al. Use of a single-balloon enteroscope compared with variable-stiffness colonoscopes for endoscopic retrograde cholangiography in liver transplant patients with Roux-en-Y biliary anastomosis. Gastrointest Endosc 2013;77(4):568-577

11 Skinner M, Popa D, Neumann H, Wilcox CM, Mönkemüller K. ERCP with the overtube-assisted enteroscopy technique: a systematic review. Endoscopy 2014;46(7):560-572

12 Samuel I, Mason EE, Renquist KE, Huang YH, Zimmerman MB, Jamal M. Bariatric surgery trends: an 18-year report from the International Bariatric Surgery Registry. Am J Surg 2006;192(5):657-662

13 Hamdan K, Somers S, Chand M. Management of late postoperative complications of bariatric surgery. $\mathrm{Br} \mathrm{J}$ Surg 2011;98(10):1345-1355

14 Hintze RE, Adler A, Veltzke W, Abou-Rebyeh H. Endoscopic access to the papilla of Vater for endoscopic retrograde cholangiopancreatography in patients with billroth II or Roux-en-Y gastrojejunostomy. Endoscopy 1997;29(2):69-73

15 Abu Dayyeh B. Single-balloon enteroscopy-assisted ERCP in patients with surgically altered GI anatomy: getting there. Gastrointest Endosc 2015;82(1):20-23 10.1016/j.gie.2015.03.1988

16 Itoi T, Ishii K, Sofuni A, et al. Large balloon dilatation following endoscopic sphincterotomy using a balloon enteroscope for the bile duct stone extractions in patients with Roux-en-Y anastomosis. Dig Liver Dis 2011;43(3):237-241

17 De Koning M, Moreels TG. Comparison of double-balloon and single-balloon enteroscope for therapeutic endoscopic retrograde cholangiography after Roux-en-Y small bowel surgery. BMC Gastroenterol 2016;16(1):9810.1186/ s12876-016-0512-6

18 Itokawa F, Itoi T, Ishii K, Sofuni A, Moriyasu F. Single- and double-balloon enteroscopy-assisted endoscopic retrograde cholangiopancreatography in patients with Roux-en-Y plus hepaticojejunostomy anastomosis and Whipple resection. Dig Endosc 2014;26(Suppl 2):136-143

19 Yane K, Katanuma A, Maguchi H, et al. Short-type single-balloon enteroscope-assisted ERCP in postsurgical altered anatomy: potential factors affecting procedural failure. Endoscopy 2017;49(1):69-74

20 Moreels TG, Kouinche Madenko N, Taha A, Piessevaux H, Deprez PH. Therapeutic enteroscopy using a new single-balloon enteroscope: a case series. Endosc Int Open 2016;4(8):E918-E921

21 Attam R, Leslie D, Freeman M, Ikramuddin S, Andrade R. EUSassisted, fluoroscopically guided gastrostomy tube placement in patients with Roux-en-Y gastric bypass: a novel technique for access to the gastric remnant. Gastrointest Endosc 2011;74(3):677-682

22 Kedia P, Kumta NA, Widmer J, et al. Endoscopic ultrasound-directed transgastric ERCP (EDGE) for Roux-en-Y anatomy: a novel technique. Endoscopy 2015;47(2):159-163

23 Bukhari M, Kowalski T, Nieto J, et al. An international, multicenter, comparative trial of EUS-guided gastrogastrostomy-assisted ERCP versus enteroscopy-assisted ERCP in patients with Roux-en-Y gastric bypass anatomy. Gastrointest Endosc 2018;88(3):486-494

24 Weber A, Gaa J, Rosca B, et al. Complications of percutaneous transhepatic biliary drainage in patients with dilated and nondilated intrahepatic bile ducts. Eur J Radiol 2009;72(3):412-417 